\title{
On The Hermite- Hadamard-Fejér Type Integral Inequality for Convex Function
}

\author{
Mehmet Zeki Sarikaya ${ }^{1, *}$, Samet Erden $^{2}$ \\ ${ }^{1}$ Department of Mathematics, Faculty of Science and Arts, Düzce University, Konu-ralp Campus, Düzce-TURKEY \\ ${ }^{2}$ Department of Mathematics, Faculty of Science, Bartn University, Konuralp Cam-pus, BARTIN-TURKEY \\ *Corresponding author: sarikayamz@gmail.com
}

Received May 14, 2014; Revised June 25, 2014; Accepted July 03, 2014

\begin{abstract}
In this paper, we extend some estimates of the right hand side of a Hermite- Hadamard-Fejér type inequality for functions whose first derivatives absolute values are convex. The results presented here would provide extensions of those given in earlier works.
\end{abstract}

Keywords: Ostrowski's inequality, Montgomery's identities, convex function, Hölder inequality

Cite This Article: Mehmet Zeki Sarikaya, and Samet Erden, "On The Hermite- Hadamard-Fejér Type Integral Inequality for Convex Function." Turkish Journal of Analysis and Number Theory, vol. 2, no. 3 (2014): 85-89. doi: 10.12691/tjant-2-3-6.

\section{Introduction}

Definition 1. The function $f:[a, b] \subset \mathbb{R} \rightarrow \mathbb{R}$, is said to be convex if the following inequality holds

$$
f(\lambda x+(1-\lambda) y) \leq \lambda f(x)+(1-\lambda) f(y)
$$

for all $\mathrm{x}, \mathrm{y} \in[\mathrm{a}, \mathrm{b}]$ and $\lambda \in[0,1]$. We say that $\mathrm{f}$ is concave if $(-\mathrm{f})$ is convex.

The following inequality is well known in the literature as the Hermite-Hadamard integral inequality (see, $[4,10])$ :

$$
f\left(\frac{a+b}{2}\right) \leq \frac{1}{b-a} \int_{a}^{b} f(x) d x \leq \frac{f(a)+f(b)}{2}
$$

where $f: I \subset \mathbb{R} \rightarrow \mathbb{R}$ is a convex function on the interval I of real numbers and $\mathrm{a}, \mathrm{b} \in \mathrm{I}$ with $\mathrm{a}<\mathrm{b}$.

In [3], Dragomir and Agarwal proved the following results connected with the right part of (1.1).

Lemma 1. Let $f: I^{\circ} \subseteq \mathbb{R} \rightarrow \mathbb{R}$ be a differentiable mapping on $\mathrm{I}^{\circ}, \mathrm{a}, \mathrm{b} \in \mathrm{I}^{\circ}$ with $\mathrm{a}<\mathrm{b}$. If $\mathrm{f}^{\prime} \in \mathrm{L}[\mathrm{a}, \mathrm{b}]$, then the following equality holds:

$$
\begin{aligned}
& \frac{f(a)+f(b)}{2}-\frac{1}{b-a} \int_{a}^{b} f(x) d x \\
& =\frac{b-a}{2} \int_{0}^{1}(1-2 t) f^{\prime}(t a+(1-t) b) d t
\end{aligned}
$$

Theorem 1. Let $f: I^{\circ} \subseteq \mathbb{R} \rightarrow \mathbb{R}$ be a differentiable mapping on $\mathrm{I}^{\circ}, \mathrm{a}, \mathrm{b} \in \mathrm{I}^{\circ}$ with $\mathrm{a}<\mathrm{b}$. If $\left|\mathrm{f}^{\prime}\right|$ is convex on $[\mathrm{a}, \mathrm{b}]$, then the following inequality holds:

$$
\begin{aligned}
& \left|\frac{f(a)+f(b)}{2}-\frac{1}{b-a} \int_{a}^{b} f(x) d x\right| \\
& \leq \frac{(b-a)}{8}\left(\left|f^{\prime}(a)\right|+\left|f^{\prime}(b)\right|\right)
\end{aligned}
$$

Theorem 2. Let $f: I^{\circ} \subseteq \mathbb{R} \rightarrow \mathbb{R}$ be a differentiable mapping on $\mathrm{I}^{\circ}, \mathrm{a}, \mathrm{b} \in \mathrm{I}^{\circ}$ with $\mathrm{a}<\mathrm{b} ; \mathrm{f}^{\prime} \in \mathrm{L}(\mathrm{a}, \mathrm{b})$ and $\mathrm{p}>1$. If the mapping $\left|\mathrm{f}^{\mathrm{p}}\right|^{\mathrm{p}(\mathrm{p}-1)}$ is convex on $[\mathrm{a}, \mathrm{b}]$, then the following inequality holds:

$$
\begin{aligned}
& \left|\frac{f(a)+f(b)}{2}-\frac{1}{b-a} \int_{a}^{b} f(x) d x\right| \\
& \leq \frac{b-a}{2(p+1)^{1 / p}}\left(\frac{\left|\mathrm{f}^{\prime}(a)\right|^{\mathrm{p} /(\mathrm{p}-1)}+\left|\mathrm{f}^{\prime}(b)\right|^{\mathrm{p} /(\mathrm{p}-1)}}{2}\right)^{(p-1) / p}(1.4)
\end{aligned}
$$

The most well-known inequalities related to the integral mean of a convex function are the Hermite Hadamard inequalities or its weighted versions, the so-called Hermite- Hadamard- Fejér inequalities (see, $[8,13,14,15,16,19,20])$. In [7], Fejer gave a weighted generalizatinon of the inequalities (1.1) as the following:

Theorem 3. $f:[a, b] \rightarrow \mathbb{R}$, be a convex function, then the inequality

$$
\begin{aligned}
& f\left(\frac{a+b}{2}\right) \int_{a}^{b} w(x) d x \leq \frac{1}{b-a} \int_{a}^{b} f(x) w(x) d x \\
& \leq \frac{f(a)+f(b)}{2} \int_{a}^{b} w(x) d x
\end{aligned}
$$

holds, where $w:[a, b] \rightarrow \mathbb{R}$ is nonnegative, integrable, and symmetric about $x=\frac{a+b}{2}$.

In [13], some inequalities of Hermite-Hadamard-Fejer type for differentiable convex mappings were proved using the following lemma.

Lemma 2. Let $f: I^{\circ} \subset \mathbb{R} \rightarrow \mathbb{R}$ be a differentiable mapping on $\mathrm{I}^{\circ}, \mathrm{a}, \mathrm{b} \in \mathrm{I}^{\circ}$ with $\mathrm{a}<\mathrm{b}$, and $\mathrm{w}:[\mathrm{a}, \mathrm{b}] \rightarrow[0, \infty)$ be $\mathrm{a}$ differentiable mapping. If $\mathrm{f}^{\prime} \in \mathrm{L}[\mathrm{a}, \mathrm{b}]$, then the following equality holds: 


$$
\begin{aligned}
& \frac{f(a)+f(b)}{2} \int_{a}^{b} w(x) d x-\int_{a}^{b} f(x) w(x) d x= \\
& \frac{(b-a)^{2}}{2} \int_{0}^{1} p(t) f^{\prime}(t a+(1-t) b) d t
\end{aligned}
$$

for each t $2[0,1]$; where

$$
p(t)=\int_{t}^{1} w(a s+(1-s) b) d s-\int_{0}^{t} w(a s+(1-s) b) d s
$$

The main result in [13] is as follows:

Theorem 4. Let $f: I^{\circ} \subset \mathbb{R} \rightarrow \mathbb{R}$ be a differentiable mapping on $\mathrm{I}^{\circ}, \mathrm{a}, \mathrm{b} \in \mathrm{I}^{\circ}$ with $\mathrm{a}<\mathrm{b}$, and $\mathrm{w}:[\mathrm{a}, \mathrm{b}] \rightarrow[0, \infty)$ be a differentiable mapping and symmetric to $\frac{a+b}{2}$. If $\left|\mathrm{f}^{\prime}\right|$ is convex on $[\mathrm{a}, \mathrm{b}]$; then the following inequality holds:

$$
\begin{aligned}
& \left|\frac{f(a)+f(b)}{2} \int_{a}^{b} w(x) d x-\int_{a}^{b} f(x) w(x) d x\right| \\
& \leq \frac{b-a}{2}\left[\int_{0}^{1}(g(t))^{p} d t\right]^{\frac{1}{p}}\left(\frac{\left|f^{\prime}(a)\right|^{q}+\left|f^{\prime}(b)^{q}\right|}{2}\right)^{\frac{1}{q}}
\end{aligned}
$$

where $g(t)=\left|\int_{a+(b-a) t}^{b-(b-a) t} w(x) d x\right|$ for $\mathrm{t} \in[0,1]$.

Definition 2. Let $\mathrm{f} \in \mathrm{L}_{1}[\mathrm{a}, \mathrm{b}]$. The Riemann-Liouville integrals $J_{a+}^{\alpha} f$ and $J_{b-}^{\alpha} f$ of order $\alpha>0$ with a $\geq 0$ are defined by

$$
J_{a+}^{\alpha} f(x)=\frac{1}{\Gamma(\alpha)} \int_{a}^{x}(x-t)^{\alpha-1} f(t) d t, x>a
$$

And

$$
J_{b^{-}}^{\alpha} f(x)=\frac{1}{\Gamma(\alpha)} \int_{a}^{x}(t-x)^{\alpha-1} f(t) d t, x>b
$$

respectively. Here, $\Gamma(\alpha)$ is the Gamma function and $J_{a+}^{0} f(x)=J_{b-}^{0} f(x)=f(x)$.

Meanwhile, Sarikaya et al. [12] presented the following important integral identity including the first-order derivative of $\mathrm{f}$ to establish many interesting HermiteHadamard type inequalities for convexity functions via Riemann-Liouville fractional integrals of the order $\alpha>0$.

Lemma 3. Let $f:[a, b] \rightarrow \mathbb{R}$ be a differentiable mapping on $(a, b)$ with $a<b$. If $\mathrm{f}^{\prime} \in \mathrm{L}[\mathrm{a}, \mathrm{b}]$, then the following equality for fractional integrals holds:

$$
\begin{aligned}
& \frac{f(a)+f(b)}{2}-\frac{\Gamma(\alpha+1)}{2(b-a)^{\alpha}}\left[J_{a+}^{\alpha} f(b)+J_{b-}^{\alpha} f(a)\right] \\
& =\frac{b-a}{2} \int_{0}^{1}\left[(1-t)^{\alpha}-t^{\alpha}\right] f^{\prime}(t a+(1-t) b) d t
\end{aligned}
$$

It is remarkable that Sarikaya et al. [12] first give the following interesting integral inequalities of HermiteHadamard type involving Riemann-Liouville fractional integrals.
Theorem 5. Let $f:[a, b] \rightarrow \mathbb{R}$ be a positive function with $0 \leq \mathrm{a}<\mathrm{b}$ and $\mathrm{f} \in \mathrm{L}_{1}[\mathrm{a}, \mathrm{b}]$. If $\mathrm{f}$ is a convex function on $[a, b]$, then the following inequalities for fractional integrals hold:

$$
\begin{aligned}
& f\left(\frac{a+b}{2}\right) \leq \frac{\Gamma(\alpha+1)}{2(b-a)^{\alpha}}\left[J_{a+}^{\alpha} f(b)+J_{b-}^{\alpha} f(a)\right] \\
& \leq \frac{f(a)+f(b)}{2}
\end{aligned}
$$

with $\alpha>0$ :

For some recent results connected with fractional integral inequalities see $[1,2,8,17,18]$.

In this article, using functions whose derivatives absolute values are convex, we obtained new inequalities of Hermite-Hadamard-Fejer type and Hermite-Hadamard type involving fractional integrals. The results presented here would provide extensions of those given in earlier works.

\section{Main Results}

We will establish some new results connected with the right-hand side of (1.5) and (1.1) involving fractional integrals used the following Lemma. Now, we give the following new Lemma for our results:

Lemma 4. Let $f: I^{\circ} \subseteq \mathbb{R} \rightarrow \mathbb{R}$ be a differentiable mapping on $\mathrm{I}^{\circ}, \mathrm{a}, \mathrm{b} \in \mathrm{I}^{\circ}$ with $\mathrm{a}<\mathrm{b}$ and let $w:[a, b] \rightarrow \mathbb{R}$. If $\mathrm{f}^{\prime}, \mathrm{w} \in \mathrm{L}[\mathrm{a}, \mathrm{b}]$, then, for all $\mathrm{x} \in[\mathrm{a}, \mathrm{b}]$, the following equality holds:

$$
\begin{aligned}
& \int_{a}^{b}\left(\int_{a}^{t} w(s) d s\right)^{\alpha} f^{\prime}(t) d t-\int_{a}^{b}\left(\int_{t}^{b} w(s) d s\right)^{\alpha} f^{\prime}(t) d t \\
& =\left(\int_{a}^{t} w(s) d s\right)^{\alpha}[f(a)+f(b)] \\
& -\alpha \int_{a}^{b}\left(\int_{a}^{t} w(s) d s\right)^{\alpha-1} w(t) f(t) d t \\
& -\alpha \int_{a}^{b}\left(\int_{t}^{b} w(s) d s\right)^{\alpha-1} w(t) f(t) d t
\end{aligned}
$$

where $\alpha>1$ :

Proof. By integration by parts, we have the following equalities:

$$
\begin{aligned}
& \int_{a}^{b}\left(\int_{a}^{t} w(s) d s\right)^{\alpha} f^{\prime}(t) d t \\
& =\left.\left(\int_{a}^{t} w(s) d s\right)^{\alpha} f(t)\right|_{a} ^{b}-\alpha \int_{a}^{b}\left(\int_{a}^{t} w(s) d s\right)^{\alpha-1} w(t) f(t) d t(2.2) \\
& =\left(\int_{a}^{b} w(s) d s\right)^{\alpha} f(b)-\alpha \int_{a}^{b}\left(\int_{a}^{t} w(s) d s\right)^{\alpha-1} w(t) f(t) d t
\end{aligned}
$$

and 


$$
\begin{aligned}
& \int_{a}^{b}\left(\int_{t}^{b} w(s) d s\right)^{\alpha} f^{\prime}(t) d t \\
& =\left.\left(\int_{t}^{b} w(s) d s\right)^{\alpha} f(t)\right|_{a} ^{b}+\alpha \int_{a}^{b}\left(\int_{t}^{b} w(s) d s\right)^{\alpha-1} w(t) f(t) d t \\
& =-\left(\int_{a}^{b} w(s) d s\right)^{\alpha} f(a)+\alpha \int_{a}^{b}\left(\int_{t}^{b} w(s) d s\right)^{\alpha-1} w(t) f(t) d t
\end{aligned}
$$

Subtracting (2.3) from (2.2), we obtain (2.1). This completes the proof.

Remark 1. If we take w(s) $=1$ in 2.1; the identity (2.1) reduces to the identity (1.8).

Corollary 1. Under the same assumptions of Lemma 4 with $\alpha=1$; then the following identity holds:

$$
\begin{aligned}
& \frac{1}{2} \int_{a}^{b}\left[\left(\int_{a}^{t} w(s) d s\right)-\left(\int_{t}^{b} w(s) d s\right)\right] f^{\prime}(t) d t \\
& =\left(\int_{a}^{b} w(s) d s\right) \frac{f(a)+f(b)}{2}-\int_{a}^{b} w(t) f(t) d t
\end{aligned}
$$

Remark 2. If we take w(s) $=1$ in (2.4), the identity (2.4) reduces to the identity (1.2).

Now, by using the above lemma, we prove our main theorems:

Theorem 6. Let $f: I^{\circ} \subseteq \mathbb{R} \rightarrow \mathbb{R}$ be a differentiable mapping on $\mathrm{I}^{\circ}, \mathrm{a}, \mathrm{b} \in \mathrm{I}^{\circ}$ with $\mathrm{a}<\mathrm{b}$ and let $w:[a, b] \rightarrow \mathbb{R}$ be continuous on $[a, b]$. If $\left|f^{\prime}\right|$ is convex on $[a, b]$, then the following inequality holds:

$$
\begin{aligned}
& \left.\mid \int_{a}^{b} w(s) d s\right)^{\alpha}[f(a)+f(b)] \\
& -\alpha \int_{a}^{b}\left(\int_{a}^{t} w(s) d s\right)^{\alpha-1} w(t) f(t) d t \\
& -\alpha \int_{a}^{b}\left(\int_{a}^{t} w(s) d s\right)^{\alpha-1} w(t) f(t) d t \mid \\
& \leq \frac{(b-a)^{\alpha+1}\|w\|_{\infty}^{\alpha}}{(\alpha+1)}\left[\left|f^{\prime}(a)\right|+\left|f^{\prime}(b)\right|\right]
\end{aligned}
$$

where $\alpha>0$ and $\|w\|_{\infty}=\sup _{t \in[a, b]}|w(t)|$.

Proof. We take absolute value of (2.1), we find that

$$
\begin{aligned}
& \mid\left(\int_{a}^{b} w(s) d s\right)^{\alpha}[f(a)+f(b)] \\
& -\alpha \int_{a}^{b}\left(\int_{a}^{t} w(s) d s\right)^{\alpha-1} w(t) f(t) d t \\
& -\alpha \int_{a}^{b}\left(\int_{t}^{b} w(s) d s\right)^{\alpha-1} w(t) f(t) d t \mid
\end{aligned}
$$

$$
\begin{aligned}
& \leq \int_{a}^{b}\left(\int_{a}^{t} w(s) d s\right)^{\alpha}\left|f^{\prime}(t)\right| d t+\int_{a}^{b}\left(\int_{t}^{b} w(s) d s\right)^{\alpha}\left|f^{\prime}(t)\right| d t \\
& \leq\|w\|_{[a, b], \infty}^{\alpha} \int_{a}^{b}(t-a)^{\alpha}\left|f^{\prime}(t)\right| d t \\
& +\|w\|_{[a, b], \infty} \int_{a}^{b}(b-t)^{\alpha}\left|f^{\prime}(t)\right| d t \\
& =\|w\|_{[a, b], \infty}^{\alpha}\left\{\int_{a}^{b}(t-a)^{\alpha}\left|f^{\prime}\left(\frac{b-t}{b-a} a+\frac{t-a}{b-a} b\right)\right| d t\right. \\
& \left.+\int_{a}^{b}(b-t)^{\alpha}\left|f^{\prime}\left(\frac{b-t}{b-a} a+\frac{t-a}{b-a} b\right)\right| d t\right\}
\end{aligned}
$$

Since $\left|\mathrm{f}^{\prime}\right|$ is convex on $[\mathrm{a}, \mathrm{b}]$, it follows that

$$
\begin{aligned}
& \mid\left(\int_{a}^{b} w(s) d s\right)^{\alpha}[f(a)+f(b)] \\
& -\alpha \int_{a}^{b}\left(\int_{a}^{t} w(s) d s\right)^{\alpha-1} w(t) f(t) d t \\
& -\alpha \int_{a}^{b}\left(\int_{t}^{b} w(s) d s\right)^{\alpha-1} w(t) f(t) d t \mid \\
& \leq\|w\|_{\infty}^{\alpha}\left\{\int_{a}^{b}(t-a)^{\alpha}\left[\frac{b-t}{b-a}\left|f^{\prime}(a)\right|+\frac{t-a}{b-a}\left|f^{\prime}(b)\right|\right] d t\right. \\
& \left.+\int_{a}^{b}(b-t)^{\alpha}\left[\frac{b-t}{b-a}\left|f^{\prime}(a)\right|+\frac{t-a}{b-a}\left|f^{\prime}(b)\right|\right] d t\right\} \\
& =\frac{(b-a)^{\alpha+1} \|\left. w\right|_{\infty} ^{\alpha}}{(\alpha+1)}\left[\left|f^{\prime}(a)+\right| f^{\prime}(b)||\right] .
\end{aligned}
$$

Hence, the proof of theorem is completed.

Corollary 2. Under the same assumptions of Theorem 6 with $\mathrm{w}(\mathrm{s})=1$, then the following inequality holds:

$$
\begin{aligned}
& \left|\frac{f(a)+f(b)}{2}-\frac{\Gamma(\alpha+1)}{2(b-a)^{\alpha}}\left[J_{a+}^{\alpha} f(b)+J_{b-}^{\alpha} f(a)\right]\right| \\
& \leq \frac{(b-a)}{(\alpha+1)}\left(\frac{\left|f^{\prime}(a)+\right| f^{\prime}(b)||}{2}\right)
\end{aligned}
$$

Proof. This proof is given by Sarikaya et. al in [11].

Remark 3. If we take $\alpha=1$ in (2.5); the inequality (2.5) reduces to (1.3).

Corollary 3. Under the same assumptions of Theorem 6 with $\alpha=1$, then the following inequality holds:

$$
\begin{aligned}
& \mid\left(\int_{a}^{b} w(s) d s\right) \frac{f(a)+f(b)}{2}-\int_{a}^{b} w(t) f(t) d t \\
\leq & \frac{(b-a)^{2}\|w\|_{\infty}}{4}\left[\left|f^{\prime}(a)\right|+\left|f^{\prime}(b)\right|\right]
\end{aligned}
$$


Theorem 7. Let $f: I^{\circ} \subseteq \mathbb{R} \rightarrow \mathbb{R}$ be a differentiable mapping on $\mathrm{I}^{\circ}, \mathrm{a}, \mathrm{b} \in \mathrm{I}^{\circ}$ with $\mathrm{a}<\mathrm{b}$ and let $w:[a, b] \rightarrow \mathbb{R}$ be continuous on $[a, b]$. If $\left|\mathrm{f}^{\prime}\right|^{\mathrm{q}}$ is convex on $[\mathrm{a}, \mathrm{b}], \mathrm{q}>1$, then the following inequality holds:

$$
\begin{aligned}
& \left.|| \int_{a}^{b} w(s) d s\right)^{\alpha}[f(a)+f(b)] \\
& -\alpha \int_{a}^{b}\left(\int_{a}^{t} w(s) d s\right)^{\alpha-1} w(t) f(t) d t \\
& -\alpha \int_{a}^{b}\left(\int_{t}^{b} w(s) d s\right)^{\alpha-1} w(t) f(t) d t \mid \\
& \leq \frac{2\|w\|_{\infty}^{\alpha}(b-a)^{\alpha+1}}{(\alpha p+1)^{\frac{1}{p}}}\left(\frac{\left|f^{\prime}(a)\right|^{q}+\left|f^{\prime}(b)\right|^{q}}{2}\right)^{\frac{1}{q}}
\end{aligned}
$$

where $\alpha>0, \frac{1}{p}+\frac{1}{q}=1$, and $\|w\|_{\infty}=\sup _{t \in[a, b]}|w(t)|$.

Proof. We take absolute value of (2.1). Using Holder's inequality, we find that

$$
\begin{aligned}
& \mid\left(\int_{a}^{b} w(s) d s\right)^{\alpha}[f(a)+f(b)] \\
& -\alpha \int_{a}^{b}\left(\int_{a}^{t} w(s) d s\right)^{\alpha-1} w(t) f(t) d t \\
& \left.-\alpha \int_{a}^{b} \mid \int_{t}^{b} w(s) d s\right)^{\alpha-1} w(t) f(t) d t \mid \\
& \leq \int_{a}^{b}\left|\int_{a}^{t} w(s) d s\right|\left|f^{\prime}(t)\right| d t+\int_{a}^{b}\left|\int_{t}^{b} w(s) d s\right|^{\alpha}\left|f^{\prime}(t)\right| d t \\
& \leq\left(\int_{a}^{b}\left|\int_{a}^{t} w(s) d s\right|^{\alpha p} d t\right)^{\frac{1}{p}}\left(\int_{a}^{b}\left|f^{\prime}(t)\right|^{q} d t\right)^{\frac{1}{q}} \\
& +\left(\left.\int_{a}^{b} \int_{t}^{b} w(s) d s\right|^{\alpha p} d t\right)^{\frac{1}{p}}\left(\int_{a}^{b}\left|f^{\prime}(t)\right|^{q} d t\right)^{\frac{1}{q}} \\
& \leq\|w\|_{\infty}^{\alpha}\left[\left(\int_{a}^{b}|t-a|^{\alpha p} d t\right)^{\frac{1}{p}}\right]\left(\left[\int_{b}^{b}\left|f^{\prime}(t)\right|^{q} d t\right)^{\frac{1}{q}}\right.
\end{aligned}
$$

Since $\left|f^{\prime}(t)\right|^{q}$ is convex on $[\mathrm{a}, \mathrm{b}]$

$$
\left|f^{\prime}\left(\frac{b-t}{b-a} a+\frac{t-a}{b-a} b\right)\right|^{q} \leq \frac{b-t}{b-a}\left|f^{\prime}(a)\right|^{q}+\frac{t-a}{b-a}\left|f^{\prime}(b)\right|^{q}(2.7)
$$

From (2.7), it follows that

$$
\begin{gathered}
\mid\left(\int_{a}^{b} w(s) d s\right)^{\alpha}[f(a)+f(b)] \\
-\alpha \int_{a}^{b}\left(\int_{a}^{t} w(s) d s\right)^{\alpha-1} w(t) f(t) d t \\
-\alpha \int_{a}^{b}\left(\int_{t}^{b} w(s) d s\right)^{\alpha-1} w(t) f(t) d t \mid \\
\leq \frac{2\|w\|_{\infty}^{\alpha}(b-a)^{\alpha+1}}{(\alpha p+1)^{\frac{1}{p}}}\left(\frac{\left|f^{\prime}(a)\right|^{q}+\left|f^{\prime}(b)\right|^{q}}{2}\right)^{\frac{1}{q}}
\end{gathered}
$$

which this completes the proof.

Corollary 4. Under the same assumptions of Theorem 6 with $\mathrm{w}(\mathrm{s})=1$, then the following inequality holds:

$$
\begin{aligned}
& \left|\frac{f(a)+f(b)}{2}-\frac{\Gamma(\alpha+1)}{2(b-a)^{\alpha}}\left[J_{a+}^{\alpha} f(b)+J_{b-}^{\alpha} f(x)\right]\right| \\
& \leq \frac{(b-a)}{(\alpha p+1)^{\frac{1}{p}}}\left(\frac{\left|f^{\prime}(a)\right|^{q}+\left|f^{\prime}(b)\right|^{q}}{2}\right)^{\frac{1}{q}}
\end{aligned}
$$

Corollary 5. Let the conditions of Theorem 7 hold. If we take $\alpha=1$ in (2.6), then the following inequality holds:

$$
\begin{aligned}
& \left|\left(\int_{a}^{b} w(s) d s\right) \frac{f(a)+f(b)}{2}-\int_{a}^{b} w(t) f(t) d t\right| \\
& \leq \frac{\|w\|_{\infty}(b-a)^{2}}{(p+1)^{\frac{1}{p}}}\left(\frac{\left|f^{\prime}(a)\right|^{q}+\left|f^{\prime}(b)\right|^{q}}{2}\right)^{\frac{1}{q}}
\end{aligned}
$$

Remark 4. If we take w(s) $=1$ in (2.9), we have

$$
\begin{aligned}
& \left|\frac{f(a)+f(b)}{2}-\frac{1}{b-a} \int_{a}^{b} f(t) d t\right| \\
& \leq \frac{(b-a)}{(p+1)^{\frac{1}{p}}}\left(\frac{\left|f^{\prime}(a)\right|^{q}+\left|f^{\prime}(b)\right|^{q}}{2}\right)^{\frac{1}{q}}
\end{aligned}
$$

which is proved by Dragomir and Agarwal in [3].

\section{References}

[1] Z. Dahmani, On Minkowski and Hermite-Hadamard integral inequalities via fractional integration, Ann. Funct. Anal. 1 (1) (2010), 51-58.

[2] J. Deng and J. Wang, Fractional Hermite-Hadamard inequalities for $(\alpha ; \mathrm{m})$-logarithmically convex functions.

[3] S. S. Dragomir and R.P. Agarwal, Two inequalities for differentiable mappings and applications to special means of real numbers and to trapezoidal formula, Appl. Math. lett., 11 (5) (1998), 91-95. 
[4] S. S. Dragomir and C. E. M. Pearce, Selected Topics on HermiteHadamard Inequalities and Applications, RGMIA Monographs, Victoria University, 2000.

[5] S. Hussain, M.A. Latif and M. Alomari, Generalized dubleintegral Ostrowski type inequalities on time scales, Appl. Math. Letters, 24 (2011), 1461-1467.

[6] M. E. Kiris and M. Z. Sarikaya, On the new generalization of Ostrowski type inequality for double integrals, International Journal of Modern Mathematical Sciences, 2014, 9 (3): 221-229.

[7] L. Fejer, Über die Fourierreihen, II. Math. Naturwiss. Anz Ungar Akad. Wiss., 24 (1906), 369.390. (Hungarian).

[8] I. I, scan, Hermite-Hadamard-Fejer type inequalities for convex functions via fractional integrals, arXiv preprint arXiv: 1404. 7722 (2014).

[9] U.S. Krmac, Inequalities for dif ferentiable mappings and applications to special means of real numbers and to midpoint formula, Appl. Math. Comp., 147 (2004), 137-146.

[10] J. Pe $\mu$ cari'c, F. Proschan and Y.L. Tong, Convex functions, partial ordering and statistical applications, Academic Press, New York, 1991.

[11] M. Z. Sarikaya, E. Set, H. Yaldiz and N., Basak, Hermite Hadamard.s inequalities for fractional integrals and related fractional inequalities, Mathematical and Computer Modelling, 57 (2013) 2403. 2407.

[12] M. Z. Sarikaya and H. Yildirim, On Hermite-Hadamard type inequalities for Riemann- Liouville fractional integrals, Submited
[13] M. Z. Sarikaya, On new Hermite Hadamard Fejer Type integral inequalities, Studia Universitatis Babes-Bolyai Mathematica., 57 (2012), No. 3, 377-386.

[14] K-L. Tseng, G-S. Yang and K-C. Hsu, Some inequalities for differentiable mappings and applications to Fejer inequality and weighted trapozidal formula, Taiwanese J. Math. 15 (4), pp: 17371747,2011

[15] C.-L. Wang, X.-H. Wang, On an extension of Hadamard inequality for convex functions, Chin. Ann. Math. 3 (1982) 567. 570.

[16] S.-H. Wu, On the weighted generalization of the HermiteHadamard inequality and its applications, The Rocky Mountain J. of Math., vol. 39, no. 5, pp. 1741. 1749, 2009.

[17] M. Tunc, On new inequalities for h-convex functions via Riemann-Liouville fractional integration, Filomat 27: 4 (2013), 559. 565.

[18] J. Wang, X. Li, M. Feckan and Y. Zhou, Hermite-Hadamard-type inequalities for Riemann-Liouville fractional integrals via two kinds of convexity, Appl. Anal. (2012).

[19] B-Y, Xi and F. Qi, Some Hermite-Hadamard type inequalities for differentiable convex func- tions and applications, Hacet. J. Math. Stat. 42 (3), 243. 257 (2013).

[20] B-Y, Xi and F. Qi, Hermite-Hadamard type inequalities for functions whose derivatives are of convexities, Nonlinear Funct. Anal. Appl. 18 (2), 163. 176 (2013).

[21] Y. Zhang and J-R. Wang, On some new Hermite-Hadamard inequalities involving Riemann-Liouville fractional integrals, Journal of Inequalities and Applications 2013, 2013: 220. 